\title{
Prepulse effects on magnitude estimation of startle-eliciting stimuli and startle responses
}

\author{
TERRY D. BLUMENTHAL, EDWARD J. SCHICATANO, JEREMY G. CHAPMAN, \\ CHRISTOPHER M. NORRIS, and EDWARD R. ERGENZINGER, JR. \\ Wake Forest University, Winston-Salem, North Carolina
}

\begin{abstract}
The present studies investigated the relationship between prepulse effects on the modification of the brainstem startle reflex and magnitude estimates of startle-eliciting stimuli. In Experiment 1, startle eyeblink responses were elicited in 24 students, half of whom were instructed to estimate the loudness of the startle stimulus (actual intensities of 80,90, and $100 \mathrm{~dB}$ ) and half of whom were instructed to estimate the magnitude of their eyeblink. When weak acoustic prepulses preceded the startle-eliciting stimulus, eyeblink amplitude was inhibited, and estimates of response magnitude decreased, but estimates of startle stimulus magnitude decreased only when 100-dB startle stimuli were presented. In Experiment 2, the same startle stimuli were preceded on some trials by a vibrotactile prepulse to the hand. In conditions in which startle amplitude was inhibited, startle stimulus magnitude estimates were not affected. This suggests that the effect of acoustic prepulses on 100-dB startle stimuli in Experiment 1 may have been due to loudness assimilation, an effect independent of the prepulse inhibition of startle responding.
\end{abstract}

The purpose of the present studies was to evaluate the effects of a prepulse on both the startle blink reflex and the estimation of the magnitude of either the startle-eliciting stimulus or the blink response itself. The startle response is a brainstem reflex to a sudden stimulus, such as a sudden sound, a flash of light, a tap to the forehead, a puff of air to the side of the face, or an electrical pulse to the forehead (Anthony, 1985). This response is very sensitive to small variations in the eliciting stimulus, and startle provides an excellent measure of sensory processing and motor excitation (Britt \& Blumenthal, 1993; Graham, 1975). The startle response can be inhibited by the presentation of a low-intensity stimulus, called a prepulse, between 30 and $500 \mathrm{msec}$ before startle stimulus onset (Graham, 1975). This startle response inhibition can be used to assess the early stages of prepulse processing. Graham (1979) has stated that inhibition of the startle response may be due to the activation of a mechanism that protects the preattentive processing of the prepulse and partially prevents this processing from being interrupted by the startle stimulus. Evidence for this protection has recently been reported (Norris \& Blumenthal, 1995).

Yamada (1983) showed that both the amplitude of the eyeblink (electromyography [EMG] of orbicularis oculi) and estimations of the magnitude of the blink-eliciting sounds increased as stimulus intensity increased from 80

The authors would like to thank E. Krauter for his assistance in the planning of these studies. Portions of this research were presented at the Psychonomic Society meeting, St. Louis, Missouri, November 1994. Correspondence should be addressed to T. D. Blumenthal, Department of Psychology, Wake Forest University, Box 7778, Reynolda Station, Winston-Salem, NC 27109 (e-mail: blumen@wfu.edu). to $120 \mathrm{~dB}$. Blumenthal (1993) presented startle-eliciting stimuli of 75,85 , and $95 \mathrm{~dB}$ and found that both blink amplitude and estimated magnitude of the startle stimulus increased with increasing stimulus intensity. Blumenthal (1993) also presented $75-\mathrm{dB}$ prepulses before $85-$ and $95-\mathrm{dB}$ startle stimuli and found that these prepulses inhibited startle but had no effect on the estimation of the loudness of the startle stimulus. Cohen, Hoffman, and Stitt (1981) assessed the effects of a prepulse on startle inhibition by measuring magnitude estimation and eyelid movement elicited by a tap to the forehead. They found that an acoustic prepulse decreased both the amount of lid movement produced by the tap and the estimated magnitude of the tap. Perlstein, Fiorito, Simons, and Graham (1993) presented pairs of acoustic stimuli (a 75-dB tone followed by a $110-\mathrm{dB}$ tone) and found that the first stimulus inhibited the startle response to the second stimulus and also decreased the estimated magnitude of the second stimulus.

Perlstein et al. (1993) also found that, for a 75-dB prepulse paired with a $110-\mathrm{dB}$ startle stimulus, the presence of the prepulse also resulted in a reduction of several scalprecorded event-related potentials (ERPs) compared to the control (no-prepulse) condition. Prepulses were associated with reductions on $\mathrm{P} 50, \mathrm{~N} 100$, and $\mathrm{P} 200$, but no effect on P30 was found. Perlstein et al. suggested that this dissociation of effects on P30 and P50 supports the contention that these two ERP components are based on different anatomical pathways, with P30 being more limited to the particular sensory system than is P50. Perlstein et al. concluded that "the cortical areas involved in early sensoriperceptual processing appear to receive in parallel at least two kinds of stimulus information: information that is independent of a preceding stimulus 
and information that is not" (p. 355), as indexed by P30 and $\mathrm{P} 50$, respectively. The cortex receives information along two paths, one attenuated by a prepulse and reflected in P50, and the other unaffected by the prepulse and reflected in P30.

Perlstein et al. (1993) provided an explanation for their observation that magnitude estimates of startle stimulus loudness are attenuated by a prepulse. They concluded that "loudness of pair members shifted toward one another" (p. 356), which would be seen as a reduction in the estimated magnitude of the startle stimulus on prepulse trials. Perlstein et al. suggested that this loudness assimilation will be present whenever the intensity difference between stimuli in a pair is above $10 \mathrm{~dB}$, but will be absent when the difference between stimulus intensities is less than $5 \mathrm{~dB}$. Because Perlstein et al. used a prepulse of $75 \mathrm{~dB}$ and a startle stimulus of $110 \mathrm{~dB}$, they had a stimulus intensity difference of $35 \mathrm{~dB}$. In the present studies, we used stimulus intensity differences greater than and less than this value, to investigate the effect of intensity difference on the effect of a prepulse on the estimate of startle stimulus magnitude. Also, given that loudness assimilation requires that both the prepulse and the startle stimulus be in the same sensory modality, we conducted a second experiment in which vibrotactile prepulses preceded acoustic startle stimuli on some trials. If both acoustic and vibrotactile prepulses attenuate startle stimulus magnitude estimates, loudness assimilation is not a sufficient explanation for this attenuation. However, if acoustic prepulses attenuate magnitude estimates and vibrotactile prepulses do not, this attenuation may be due to loudness assimilation and may be independent of the inhibition of the startle response caused by the prepulse.

The present study also included a group of subjects who were told to estimate the magnitude of their eyeblink response to the startle-eliciting stimuli. In this way, the effects of prepulses on estimates of both the startleeliciting stimulus and the resulting startle response could be measured. If the prepulse affects estimates of response magnitude but not estimates of stimulus magnitude, the absence of prepulse effects on stimulus magnitude estimation cannot be due to an inability of the subjects to execute a simple magnitude estimation task.

\section{EXPERIMENT 1}

\footnotetext{
Method

Subjects. The subjects were 24 undergraduate psychology students (age range: 17.5-20.5 years), randomly assigned to one of two groups, estimate stimulus ( $n=12 ; 6$ males) or estimate response ( $n=12 ; 6$ males). All subjects recejved class credit for their participation.

Stimuli. Startle stimuli were $80-, 90-$, and $100-\mathrm{dB}$ (A) broadband noise bursts, with a duration of $50 \mathrm{msec}$ and a rise/fall time of $0.1 \mathrm{msec}$. Prepulse stimuli were $1000-\mathrm{Hz}$ tones, with intensities of 60 and $70 \mathrm{~dB}$ (A), a duration of $20 \mathrm{msec}$, and a rise/fall time of $5 \mathrm{msec}$, presented at a stimulus onset asynchrony of $150 \mathrm{msec}$ relative to the startle stimuli. There was a total of 63 trials presented
}

to each subject, with 7 blocks of 9 trials each. In each block, each startle stimulus was presented alone once, and paired with each prepulse once, and the order of these nine conditions was random within each block. The average intertrial interval was $30 \mathrm{sec}$ (range: $25-35 \mathrm{sec}$ ).

Apparatus. Startle stimuli at each of the three intensities were produced by separate Coulbourn S81-02 noise generators gated through separate Coulbourn S84-04 rise/fall gates and amplified by a Coulbourn S82-24 audio mixer-amplifier. Tone prepulse stimuli at each of the two intensities were produced by separate Coulbourn S8 1-06 precision signal generators gated through separate Coulbourn S84-04 rise/fall gates and amplified by a Coulbourn $\$ 82-24$ audio mixer-amplifier. All stimuli were presented to the subjects through Telephonics TDH-49P headphones. The stimulus intensities were calibrated with a Quest sound level meter and headphone coupler during the presentation of a continuous stimulus, and a Fluke $8050 \mathrm{~A}$ digital multimeter was used to measure the voltage of the input to the headphones.

The reflex eyeblink responses were measured as periorbital EMG activity from orbicularis oculi, collected from SensorMedics biopotential electrodes ( $\mathrm{Ag} / \mathrm{AgCl}, 4-\mathrm{mm}$-diameter contact surface). Electrode impedance was measured with a Grass EZM5B electrode impedance meter. The EMG activity was amplified with a Coulbourn S75-01 high-gain bioamplifier, with amplification set at 5000 and filters passing EMG activity between 90 and $250 \mathrm{~Hz}$ to a Coulbourn S76-01 contour-following integrator with a $10-\mathrm{msec}$ time constant. The output of this integrator was amplified by a Coulbourn S79-02 adjustable gain amplifier. The integrated EMG signal was then converted from analog-to-digital units ( 1,000 samples per second) to microvolts and plotted as a function of time for $500 \mathrm{msec}$, beginning $150 \mathrm{msec}$ before startle stimulus onset, by a MacPacq MP 0 interface controlled by a Macintosh SE computer. An Archer two-station intercom allowed subjects to communicate their magnitude estimations to the experimenter.

Procedure. Subjects were asked to complete an informed consent form and a questionnaire inquiring about their recent health. The experimenter then cleaned the area of the face below the subject's left cye with a cotton swab dipped in rubbing alcohol. Two electrodes were placed on the skin below the left eye, one directly below the pupil, and the other just temporal to the first, with the center of the electrodes about $13 \mathrm{~mm}$ apart. A ground electrode was placed on the medial surface of the subject's left forearm, just below the elbow. The subject was seated in a sound-attenuated chamber, the impedance of the electrodes was measured, the subject was told to look forward and sit as still as possible, and the experimenter placed the headphones on the subject. The experimenter then presented different examples of the stimuli until the subject was sure that he/she could discriminate the startle stimulus from the prepulse. The experimenter then gave the subjects in the estimate stimulus group the following instructions:

You will be hearing noise bursts over the headphones, and you will be asked to estimate the loudness of these noises by assigning arbitrary values to them. Some trials will have only one noise, while others will have two noises very close together. When there are two noises together, it is very important that you estimate the loudness of the second noise, not the loudness of the first noise or a combination of the two noises. You can assign any value you want to the noises; there are no right or wrong answers. Just call out your number right after you hear the loud noise burst.

The subjects in the estimate response group received the following instructions:

You will be hearing noise bursts over the headphones. Some trials will have one noise burst, and other trials might have two sounds. The loud noises might make you blink. When you blink, you will notice some muscle activity below your eyes. We are interested in what you estimate the size of this muscle activity to be, so assign an arbitrary value to this muscle activity. This muscle may be activated even when the 
eyelid does not close, so estimate the amount of muscle activity, not the amount of eyelid closure. You can assign any value you want to the blink; there are no right or wrong answers. Also remember that zero is an acceptable value. Just call out your number right after you hear the loud noise burst.

Any questions regarding the estimation procedure were then answered, and the subjects were presented with the 63 trials of stimuli. Note that no standard stimulus was presented; subjects were free to assign any numerical value they chose, and these values were recorded on each trial by an experimenter. The reason for this is that the slope of the psychophysical function relating stimulus intensity and magnitude estimates can be affected by the choice of a standard stimulus (Engen, 1972; Gescheider, 1985). Because no standard stimulus was presented, the geometric means of the magnitude estimates in each condition were used in the data analyses (Gescheider, 1985).

Data analysis. Dependent variables were startle response amplitude, probability, latency, and magnitude estimate. Response amplitude was scored as the difference between the onset and peak of the response and was measured in arbitrary analog-to-digital convertor units during a window of 20 to $100 \mathrm{msec}$ after the onset of the startle stimulus. These values were then converted to microvolts of response amplitude based on a conversion factor arrived at through calibration.

Response latency was scored as the time between startle stimulus onset and response onset and was measured in milliseconds. Response probability was scored as the ratio, for each stimulus condition, of the number of trials on which a startle response was measured to the total number of trials on which a startle stimulus was presented and a response could have been recorded (eliminating trials with large movement artifact). Magnitude estimates were recorded on each trial, and the geometric means of the magnitude estimates for each stimulus condition were compared. Statistical analyses were conducted with analysis of variance (ANOVA, BMDP4V), with conservative Greenhouse-Geisser degrees of freedom used and epsilon values reported. Analyses of orthogonal trends were conducted with BMDP2V ANOVA. For each dependent measure, three different types of analyses were conducted. First, data in the control (no prepulse) conditions were analyzed, with startle stimulus intensity $(80,90$, and $100 \mathrm{~dB}[\mathrm{~A}])$ as a within-subjects variable and group (estimate stimulus and estimate response) as a between-subjects variable. Second, the effects of the prepulses were evaluated by comparing responding in each prepulse condition to that in the appropriate control condition, with group as a between-subjects variable. Third, the effects of prepulse intensity and startle stimulus intensity on prepulse modification of startle were analyzed, first by subtracting the response value in the prepulse condition from that in the appropriate control condition, and then by analyzing these differences with two within-subjects variables of prepulse intensity ( 60 and $70 \mathrm{~dB}[\mathrm{~A}])$ and startle stimulus intensity $(80,90$, and $100 \mathrm{~dB}[\mathrm{~A}])$, and a between-subjects variable of group (estimate stimulus and estimate response). Data are presented in the figures as the response level in each control and prepulse condition, but it should be remembered that the effects of the prepulses were tested by assessing their ability to cause responding to differ from that in the control conditions.

\section{Results}

Control (no prepulse) trials. On control trials, increasing startle stimulus intensity caused increased startle response amplitude $[F(2,44)=23.99, p<.001, \varepsilon=$ 61] (Figures 1A-1B), increased startle response probability $[F(2,44)=16.31, p<.001, \varepsilon=.59]$ (Figures $2 \mathrm{~A}$ $2 \mathrm{~B})$, decreased startle response latency $[F(2,44)=$ $16.64, p<.001, \varepsilon=.97$ ] (Figures $3 \mathrm{~A}-3 \mathrm{~B}$ ), and increased magnitude estimates $[F(2,44)=132.88, p<.001, \varepsilon=$ .67] (Figures 4A-4B). For startle response amplitude and probability, the main effect of group was not significant, nor was the group $\times$ startle stimulus intensity interaction. For response latency, a main effect of group was found $[F(1,22)=4.59, p<.05]$, as was a group $\times$ startle stimulus intensity interaction $[F(2,44)=3.52, p<.05$, $\varepsilon=.97]$ (Figures 3A-3B). Response latency was not significantly different at intensities of 80 or $90 \mathrm{~dB}$ for either group. However, increasing startle stimulus intensity from 90 to $100 \mathrm{~dB}$ caused a reduction in latency in both groups $[F(1,22)=21.05, p<.001]$, and startle latencies in this comparison were significantly faster in the estimate stimulus group than in the estimate response group $[F(1,22)=7.62, p<.025]$. Another way to describe this interaction is that response latency was significantly faster for the estimate stimulus group than for the estimate response group when startle stimulus intensity was $100 \mathrm{~dB}[F(1,22)=9.96, p<.01]$; this difference was marginally significant at $90 \mathrm{~dB}[F(1,22)=4.18, p<$ $.054]$, and no group difference was present at $80 \mathrm{~dB}$.

For magnitude estimates, a main effect of group was found $[F(1,22)=20.46, p<.001]$ (see Figures 4A-4B). Pairwise comparisons showed that estimates were larger in the estimate stimulus group than in the estimate response group at all three startle stimulus intensities. This difference may have been due to either intersubject differences in scales used for the estimates or to the presence of zero as an acceptable value for the response estimate. The option of using zero to estimate response magnitude was necessary because the startle response is probabilistic and does not occur on every trial, whereas the stimulus was presented on every trial, so that zero was not an acceptable value for stimulus magnitude estimates. However, when the magnitude estimate data were reanalyzed with zero estimates treated as missing values, the magnitude estimates were still significantly different for the two groups, and none of the statistical conclusions were affected when zero estimates were excluded for the estimate response group. Therefore, the group difference in magnitude estimates was not due to the inclusion of zero as an option in the estimate response group.

Prepulse effects. The influence of the prepulse was calculated by comparing responding in a single prepulse condition to responding in the appropriate control condition. Prepulses inhibited startle response amplitude in all conditions $(p<.01)$, and this inhibition was not different for the two groups (see Figures 1A-1B). The amount of inhibition increased with both prepulse intensity $[F(1,22)=13.53, p<.01]$ and startle stimulus intensity $[F(1,22)=3.85, p<.05, \varepsilon=.95]$. Prepulses inhibited response probability in all cases $(p<.025)$, except when $60-\mathrm{dB}$ prepulses were paired with $100-\mathrm{dB}$ startle stimuli $(p<.065)$. Inhibition of response probability was not different for the two groups (see Figures $2 \mathrm{~A}-2 \mathrm{~B}$ ). The amount of inhibition of response probability increased with both prepulse intensity $[F(1,22)=9.81, p<.01]$ and startle stimulus intensity $[F(1,22)=5.29, p<.05$, 
$\varepsilon=.87]$. Startle response latency was facilitated (reduced latency, or faster responding) by $60-\mathrm{dB}$ prepulses paired with either $80-$ or $90-\mathrm{dB}$ startle stimuli in both groups, but $60-\mathrm{dB}$ prepulses paired with $100-\mathrm{dB}$ startle stimuli, and 70-dB prepulses paired with any startle stimulus, had no effect on response latency (see Figures $3 \mathrm{~A}-3 \mathrm{~B})$.

The effect of prepulses on magnitude estimates was greater for the estimate response group than for the estimate stimulus group $[F(1,22)=41.16, p<.001]$ (see Figures 4A-4B). Prepulses paired with startle stimuli caused lower magnitude estimates in the estimate response group in all cases $(p<.001)$. In the estimate stimulus group, prepulses had no effect on magnitude estimates when startle stimuli were at either 80 or $90 \mathrm{~dB}$. However, when startle stimuli were $100 \mathrm{~dB}$, both the $60-\mathrm{dB}$ prepulses and the $70-\mathrm{dB}$ prepulses significantly decreased magnitude estimates in this group $[F(1,11)=8.13$ and 7.18 , respectively, $p<.025]$.

\section{Discussion}

In both groups of subjects in the present experiment, the effects of stimulus intensity on the elicitation of the startle response were similar, with more intense stimuli eliciting larger, faster, and more probable responses. The effects of stimulus intensity on the inhibition of startle responding by a prepulse were also the same in the two groups, with more intense prepulses causing more inhibition of startle response amplitude and probability. Also, prepulses caused greater inhibition when startle stimuli were more intense. These are all common findings in the area of startle research (Anthony, 1985; Blumenthal, 1988, 1994; Blumenthal \& Gescheider, 1987). Another interesting finding was that startle response latency was faster when subjects estimated the stimulus than when they estimated the response, most notably for high-intensity stimuli. This is akin to an attentional direction effect, in that response latency was facilitated by the direction of attention to, relative to away from, the eliciting stimulus. Facilitation of startle by directed attention has also been shown in previous research (Bohlin \& Graham, 1977; Bohlin, Graham, Silverstein, \& Hackley, 1981; Silverstein, Graham, \& Bohlin, 1981).

In both estimation task groups in the present experiment, increasing the intensity of the startle stimulus caused an increase in the magnitude estimates on control trials. These data support the findings of Blumenthal (1993) and Yamada (1983), both of whom found that increasing stimulus intensity results in larger blinks and higher stimulus magnitude estimates. This shows that the subjects were able to perform the task of magnitude estimation in a sensitive enough manner to have stimulus manipulations reflected in the magnitude estimates. Prepulses in the present experiment had no significant effect on estimates of stimulus magnitude when startle stimuli were below $100 \mathrm{~dB}$, which is similar to the findings of Blumenthal (1993). When 100-dB startle stimuli were used, stimulus magnitude estimates were significantly lower in the prepulse conditions than in the control condition. However, prepulses significantly attenuated estimates of response magnitude at all startle stimulus intensities.

Given the fact that prepulses influenced stimulus magnitude estimates in some conditions and not in others, several points must be considered. First, it might be argued that the subjects in the estimate stimulus group heard the $80-$ and $90-\mathrm{dB}$ startle stimuli as less loud on prepulse trials but were not able to provide sensitive enough estimates to reach statistical significance. These arguments are weakened by the fact that these subjects had no problem in assigning different estimates to the startle stimuli on control trials. With regard to statistical significance, the range of estimate values was considerably more narrow in the estimate response group, yet this group showed a significant effect of prepulses on response magnitude estimates at all startle stimulus intensities. The prepulses seem to have had no effect whatsoever on magnitude estimates in the estimate stimulus group when startle stimulus intensity was 80 or $90 \mathrm{~dB}$.

Second, it might be argued that subjects in the estimate stimulus group misunderstood the task and estimated the magnitude of the prepulse, or some combination of the prepulse and startle stimulus. Once again, this is unlikely, because one would then expect the magnitude estimates in the prepulse conditions to have been consistently lower than those on the control trials, and that was not the case.

Third, it might be argued that the presentation of the prepulses in some conditions was deficient in some way, due to a technical or equipment malfunction, so that the prepulses were either accidentally not presented or were too low in intensity to be perceived. The effectiveness of these prepulses as inhibitors of the startle blink reflex, however, suggests otherwise. It is clear that these prepulses influenced responding at some level, as shown by the startle inhibition data.

Other researchers have shown that prepulses that inhibit startle responding also reduce the estimated magnitude of the startle-eliciting stimulus (Cohen et al., 1981; Perlstein et al., 1993). There are several differences between those studies and the present study, but the most relevant differences have to do with the magnitude estimation task and the startle stimuli used. Both Cohen et al. and Perlstein et al. provided their subjects with a modulus, referred to as an "anchor" stimulus. This involves presenting a stimulus and telling the subject that this stimulus has a particular value (both Cohen et al. and Perlstein et al. used the value of 10 for this anchor stimulus), and this is often done to reduce the range of estimate values given by subjects. The subject is then told that stimuli that are judged to be twice as intense as the standard should be assigned a value of 20 , and those half as intense should be assigned a value of 5 .

A problem that can arise with the assignment of a modulus is that choice of a standard stimulus can affect the slope of the psychophysical function relating stimulus intensity and magnitude estimates. Specifically, when 
a standard stimulus in the middle of the intensity range is used, this slope will be highest and will decrease as the standard stimulus deviates from this middle value. Therefore, some psychophysicists recommend that a modulus not be given, and that subjects be allowed to use any numbers they choose (Engen, 1972; Gescheider, 1985). Given that both Cohen et al. (1981) and Perlstein et al. (1993) used a standard stimulus near the middle of their stimulus intensity range (14-V tap for Cohen et al.; 90$\mathrm{dB}$ sound for Perlstein et al.), this may not have had a major impact on the outcome. However, no modulus was provided in the present study, and subjects were free to use whatever scale they chose for their magnitude estimates.

Another alternative hypothesis involves response feedback from the eyeblink elicited by the intense startle stimuli. Subjects who are told to estimate the magnitude of the startle stimulus may, when presented with a very intense startle stimulus, inadvertently use their response to the stimulus as a guide in estimating stimulus magnitude. ${ }^{1}$ Response feedback may be so great in this condition as to overshadow or confuse estimates of eliciting stimulus magnitude. When the prepulse inhibits the response to the startle stimulus, the estimated magnitude of the stimulus will be contaminated by less response feedback. This might explain why effects of prepulses on startle stimulus estimation are limited to very high intensity startle stimuli.

Perlstein et al. (1993) used acoustic prepulses of $75 \mathrm{~dB}$ and startle stimuli of 75 and $110 \mathrm{~dB}$, showing decreased magnitude estimates of the more intense startle stimulus when these were preceded by a prepulse. Our data show an effect of prepulses on magnitude estimation of a 100 $\mathrm{dB}$ startle stimulus, supporting Perlstein et al.'s conclusion that the difference in intensity between the prepulse and the startle stimulus must pass some threshold before the estimation of startle stimulus magnitude is affected by the prepulse. Perlstein et al. believed that this intensity difference was $10 \mathrm{~dB}$ or greater, but the present study suggests that a minimum difference of $30 \mathrm{~dB}$ is required. That is, if the difference in intensity of the prepulse and startle stimulus is $35 \mathrm{~dB}$ (as in Perlstein et al., 1993) or $30-40 \mathrm{~dB}$ (as in the present study), the presence of the prepulse reduces the estimated magnitude of the startle stimulus. At intensity differences of less than $30 \mathrm{~dB}$, no such effect on stimulus magnitude estimates is found. This conclusion must be qualified by the fact that we found no effect of a $60-\mathrm{dB}$ prepulse on the magnitude estimates of a 90-dB startle stimulus, suggesting that an intensity difference of $30 \mathrm{~dB}$ is not the only important parameter. The requirement for a minimum difference between stimulus intensities may interact with the intensity of the louder stimulus. This might suggest that the prepulse has some effect on the sensory impact of the startle stimulus through loudness assimilation (Elmasian, Galambos, \& Bernheim, 1980), but only for very intense startle stimuli. Loudness assimilation refers to the fact that magnitude estimates of individual stimuli in a pair tend to shift toward the average loudness of the two stimuli. This would lead to startle stimuli being judged to be of lower magnitude when preceded by a prepulse, as shown by Perlstein et al. (1993) and in the present study when startle stimulus intensity was $100 \mathrm{~dB}$, but not at lower intensities. Perlstein et al. (1993) also found that estimates of prepulse magnitude increased when the prepulse was followed by a more intense stimulus, another demonstration of loudness assimilation.

One way to avoid loudness assimilation is to use a prepulse in a sensory modality different from that in which the startle stimulus is presented. Blumenthal and $\mathrm{Ge}-$ scheider (1987) showed that a brief vibration to the hand can act as an effective prepulse, modifying the acoustic startle response in a predictable fashion. Given the availability of this cross-modal effect, a second experiment was conducted, in which acoustic startle stimuli at three intensities were preceded on some trials by vibrotactile prepulses presented to the hand, at two different intensities, and subjects were told to estimate the intensity of the acoustic startle stimulus. If the effect of prepulses on the estimation of the magnitude of 100-dB startle stimuli in Experiment 1 was due to loudness assimilation, we predicted that there should be no effect of vibrotactile prepulses on startle stimulus estimates in this second experiment.

\section{EXPERIMENT 2}

\section{Method}

Subjects. The subjects were 11 undergraduate psychology students (age range: 18.2-20.5 years), selected in the same way as were the subjects in Experiment 1. Only subjects who scored as strongly right handed on a handedness questionnaire (Dorthe, Blumenthal, Jason, \& Lantz, 1995) were included in this study. No subject participated in both experiments.

Stimuli, Apparatus, and Procedure. Startle stimuli were 80-, $90-$, and $100-\mathrm{dB}$ (A) broadband noise bursts, with a duration of $50 \mathrm{msec}$ and a rise/fall time of $0.1 \mathrm{msec}$. Prepulse stimuli were $100-\mathrm{Hz}$ vibrations, with a duration of $50 \mathrm{msec}$ and a rise/fall time of $5 \mathrm{msec}$, presented at a stimulus onset asynchrony of $150 \mathrm{msec}$ relative to the startle stimuli. Prepulse intensities were crossmodality matched by having 5 observers, none of whom served as subjects for this study, adjust the perceived intensity of a constant vibration to match the loudness of a constant broadband noise at 60 and $70 \mathrm{~dB}(\mathrm{~A})$. This was done in an attempt to make the prepulse stimuli in this second experiment subjectively equivalent in intensity to the prepulses used in Experiment 1 . There were a total of 63 trials presented to each subject, with 7 blocks of 9 trials each. In each block, each startle stimulus was presented alone once, and paired with each prepulse once, and the order of these nine conditions was random within each block. The average intertrial interval was $30 \mathrm{sec}$ (range: $25-35 \mathrm{sec}$ ).

Startle stimuli were produced and presented in the same way as in Experiment 1. Vibrotactile prepulses were produced by the same tone generators as those used in Experiment 1, gated through the same rise/fall gates and audio mixer-amplifier, and presented to the subject via a Ling 203 Shaker (vibrator) with an attached 3.0$\mathrm{cm}^{2}$ contoured circular contactor, which protruded through a small hole in the desk at which the subject sat. The subject was asked to rest the right hand on the desk through which the vibrator contactor protruded. The positioning of the arm was such that the contoured disk contacted the thenar eminence (the fleshy part of the palm just below the thumb) of the right hand. The subject was asked to relax the hand and not to move it until the end of the session. The vibrator made a slight noise, but this was not detectable 
when the subject wore the headphones and the vibrator was covered with the hand. The reflex eyeblink responses and stimulus magnitude estimates were measured, quantified, and analyzed in the same way as in Experiment 1.

\section{Results}

Control (no prepulse) trials. On control trials, increasing startle stimulus intensity caused increased startle response amplitude $[F(2,20)=11.66, p<.001, \varepsilon=$ .86] (Figure $1 \mathrm{C}$ ), increased startle response probability $[F(2,20)=8.69, p<.001, \varepsilon=.79]$ (Figure 2C), decreased startle response latency $[F(2,20)=7.52, p<.01$, $\varepsilon=.75$ ] (Figure $3 \mathrm{C}$ ), and increased magnitude estimates $[F(2,20)=59.65, p<.001, \varepsilon=.61]$ (Figure 4C).

Prepulse effects. The influence of the prepulse was calculated via comparison of responding in a single pre-
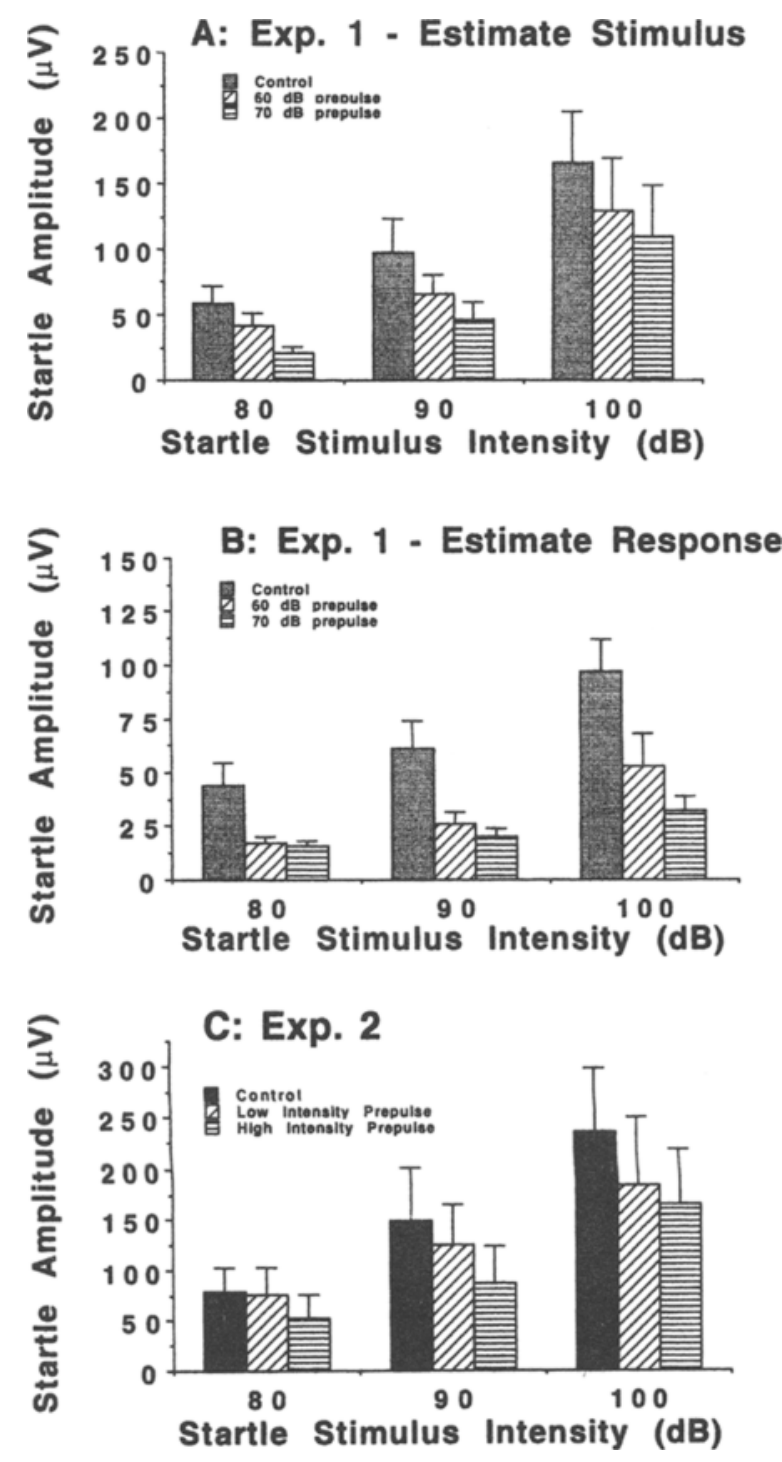

Figure 1. Startle eyeblink amplitude as a function of startle stimulus intensity and prepulse intensity in the estimate stimulus group (A) and the estimate response group (B) in Experiment 1, and in Experiment 2 (C). Lines represent 1 standard error of the mean.
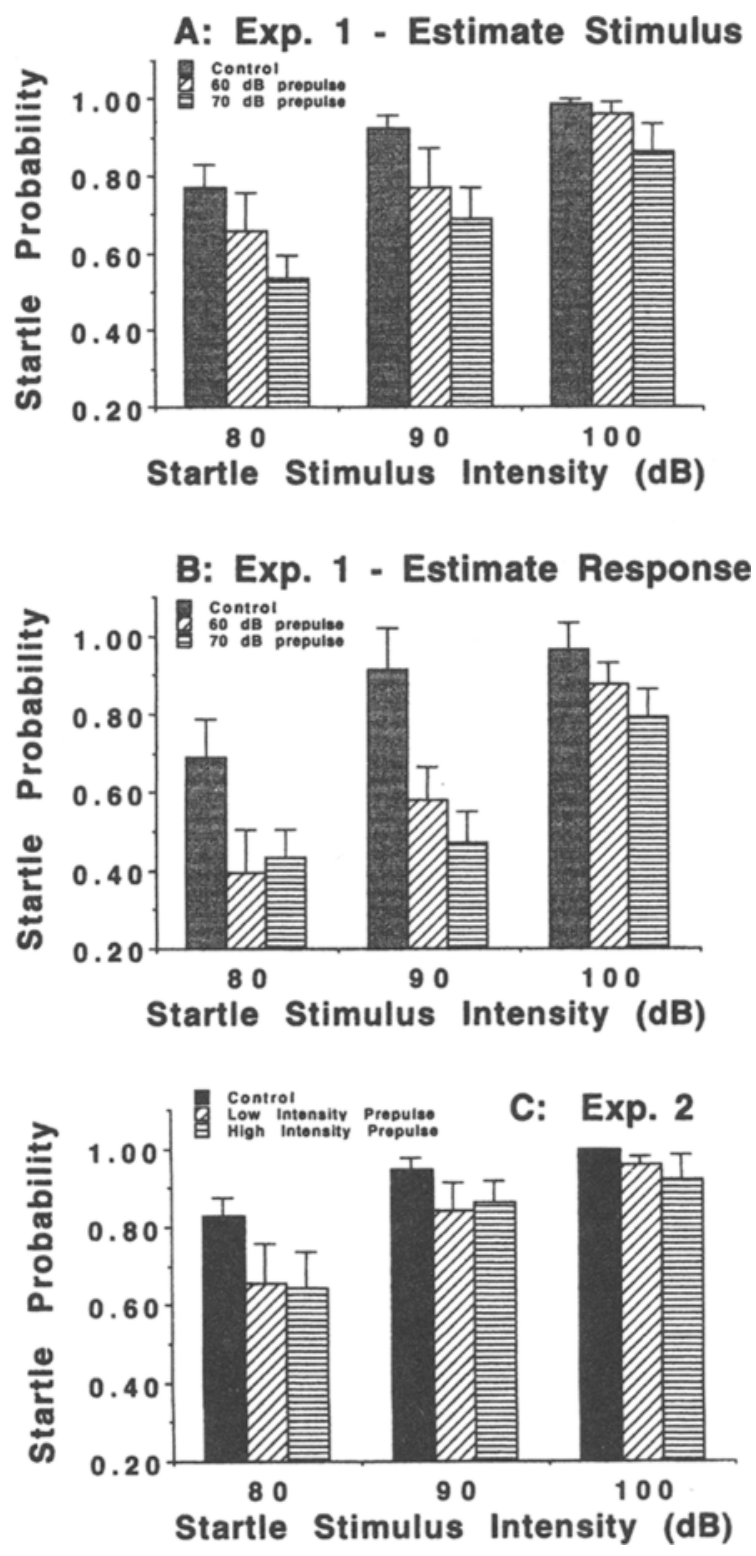

Figure 2. Startle eyeblink probability as a function of startie stimulus intensity and prepulse intensity in the estimate stimulus group (A) and the estimate response group (B) in Experiment 1, and in Experiment 2 (C). Lines represent 1 standard error of the mean.

pulse condition to responding in the appropriate control condition. The low-intensity prepulse inhibited startle amplitude only when paired with the 100-dB startle stimuli, but high-intensity prepulses inhibited startle amplitude at all three startle stimulus intensities $(p<.05)$ (Figure 1C). The amount of inhibition increased with prepulse intensity $[F(1,10)=5.06, p<.05]$ but was unaffected by startle stimulus intensity. Prepulses inhibited response probability only when the startle stimulus intensity was $80 \mathrm{~dB}(p<.05)$ (Figure $2 \mathrm{C})$. Startle response latency was facilitated (reduced latency, or faster responding) by prepulses at both intensities paired with $80-\mathrm{dB}$ startle stimuli and by the more intense prepulse 

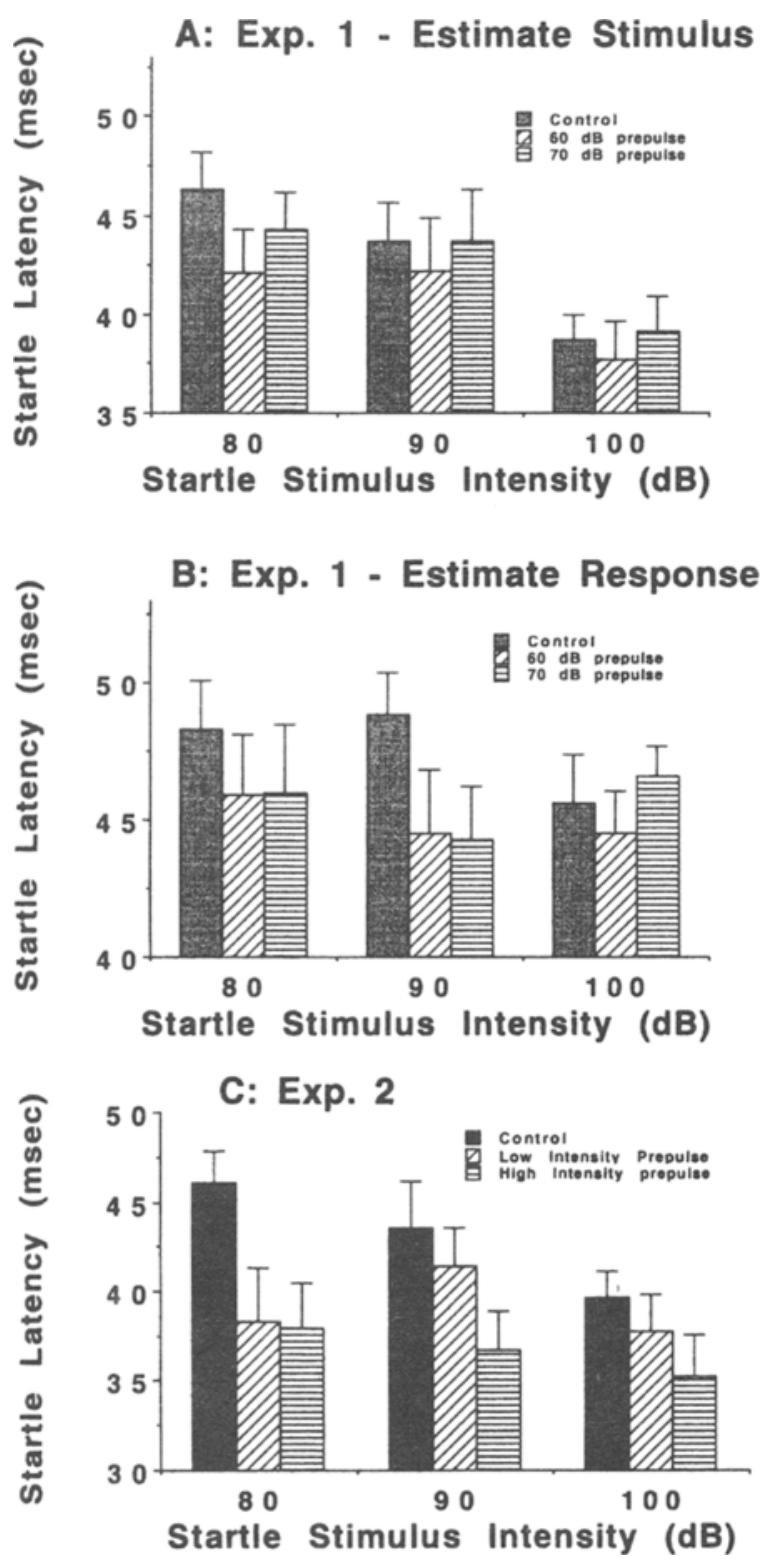

Figure 3. Startle eyeblink latency as a function of startle stimulus intensity and prepulse intensity in the estimate stimulus group (A) and the estimate response group (B) in Experiment 1, and in Experiment $2(C)$. Lines represent 1 standard error of the mean.

paired with 90-dB startle stimuli (Figure 3C). Prepulses had no effect on estimates of startle stimulus magnitude at any pairing of prepulse intensity and startle stimulus intensity (Figure 4C).

\section{Discussion}

The vibrotactile prepulses used in this experiment inhibited startle amplitude in some conditions, but the prepulse was not sufficiently intense to inhibit startle in all conditions. Blumenthal and Gescheider (1987) have shown that the amount of inhibition of startle increases as vibrotactile prepulse intensity increases, a conclusion supported by the present study. These vibrotactile pre- pulses had no impact on magnitude estimates of the startle stimuli, even at the highest startle stimulus intensity. This suggests that the influence of acoustic prepulses on 100-dB startle stimulus intensity estimates seen in Experiment 1 may have been due to loudness assimilation and may not be related to the mechanism responsible for prepulse inhibition of the startle response.

\section{GENERAL DISCUSSION}

Two types of stimulus effectiveness were assessed in this experiment: the brainstem startle reflex and the cortical estimation of stimulus and response magnitude. The startle response was affected by eliciting stimulus inten-
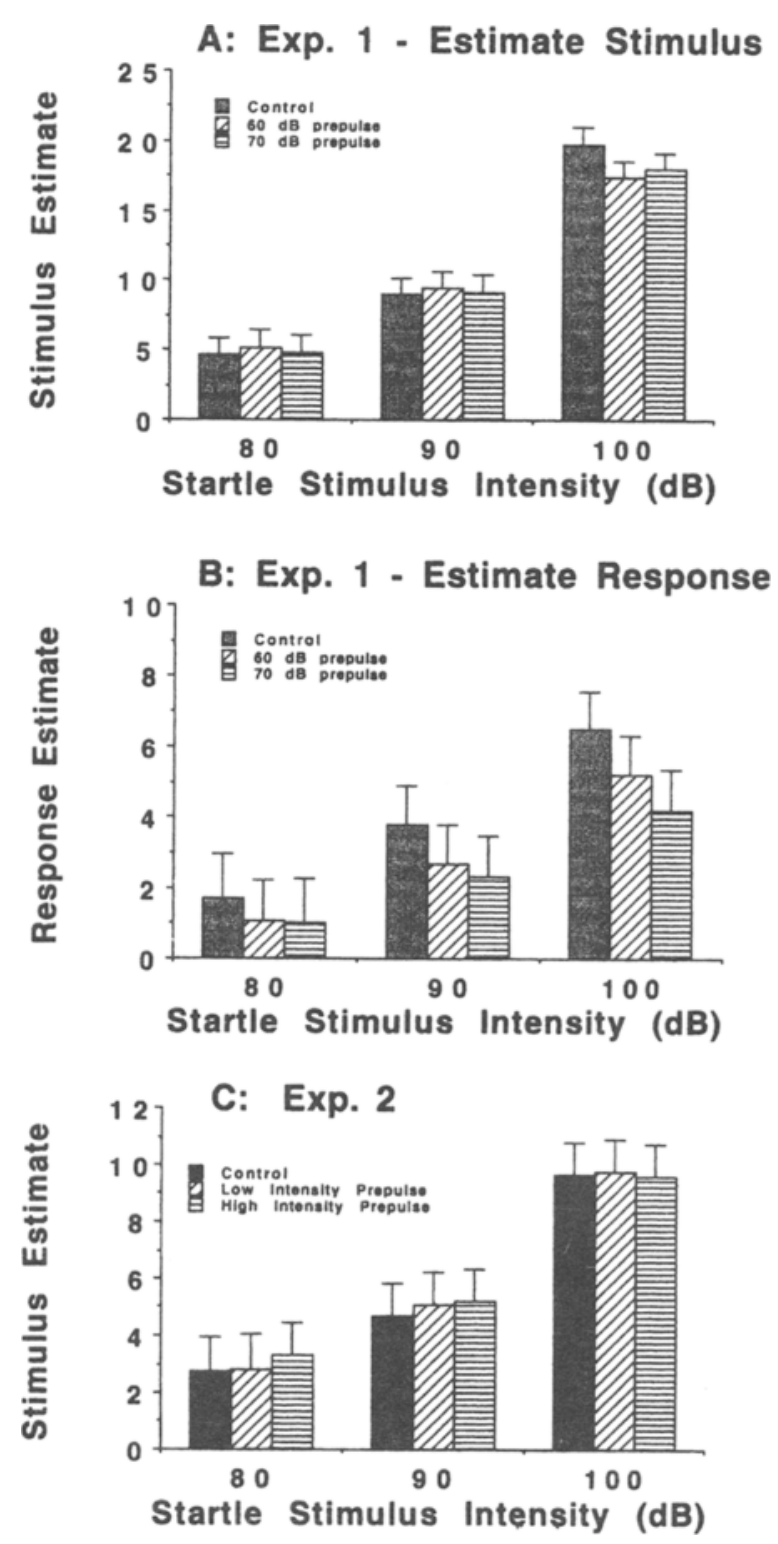

Figure 4. Magnitude estimates as a function of startle stimulus intensity and prepulse intensity in the estimate stimulus group (A) and the estimate response group (B) in Experiment 1 , and in Experiment 2 (C). Lines represent 1 standard error of the mean. 
sity, as were the estimates of the magnitude of the startle stimulus and response in control conditions. However, the two types of measurement diverged when a prepulse was presented. The prepulse clearly inhibited startle response amplitude. When subjects were told to estimate the loudness of the startle stimulus, this magnitude estimation was affected only when the acoustic prepulses were paired with the most intense startle stimuli. However, magnitude estimates did accurately reflect inhibited startle blinks in the estimate response group. This suggests that the mechanism that is activated by the prepulse to inhibit the startle response does not necessarily impair higher processing of the startle stimulus, so that the sensory impact of the startle stimulus is maintained. This is similar to the finding of Perlstein et al. (1993) of no effect of a prepulse on the P30 ERP. However, Perlstein et al. showed that other ERP components were affected by a prepulse, as were magnitude estimates of startle stimulus intensity. Our data agree with theirs to a certain extent in that they show that magnitude estimates of startle stimuli are affected by prepulses when the intensity difference between the prepulse and the startle stimulus is at least $30 \mathrm{~dB}$. However, our data show that, for intensity differences below $30 \mathrm{~dB}$, the prepulse has no effect on the estimate of the magnitude of the startle stimulus. Also, when the prepulse and startle stimulus are in different sensory modalities, the two are independent, with no prepulse effect on startle stimulus magnitude estimates. This points to loudness assimilation as a possible explanation for the findings of Perlstein et al. (1993) and for the findings in the 100-dB condition of our Experiment 1 . This also suggests that prepulse effects on magnitude estimates of startle stimuli are independent of prepulse effects on startle responding.

\section{REFERENCES}

ANTHONY, B. J. (1985). In the blink of an eye: Implications of reflex modification for information processing. In P. K. Ackles, J. R. Jennings, \& M. G. H. Coles (Eds.), Advances in psychophysiology (Vol. 1, pp. 167-218). Greenwich, CT: JAI Press.

BLumentHal, T. D. (1988). The startle response to acoustic stimuli near startle threshold: Effects of stimulus rise and fall time, duration, and intensity. Psychophysiology, 25, 607-611.

Blumenthal, T. D. (1993, March). Startle reflex modification and magnitude estimation. Paper presented at the annual meeting of the Southeastern Psychological Association, Atlanta.

Blumenthal, T. D. (1994). Prepulse inhibition is influenced by both prepulse intensity and startle stimulus intensity. Psychophysiology, 31, S27.

Blumenthal, T. D., \& Gescheider, G. A. (1987). Modification of the acoustic startle response by a tactile prepulse: Effects of stimulus onset asynchrony and prepulse intensity. Psychophysiology, 24, 320327.

Bohlin, G, \& Graham, F. K. (1977). Cardiac deceleration and reflex blink facilitation. Psychophysiology, 14, 423-430.

Bohlin, G., Graham, F. K., Silverstein, L. D., \& Hackley, S. A. (1981). Cardiac orienting and startle blink modification in novel and signal situations. Psychophysiology, 18, 603-611.

BRITT, T. W., \& BLUMENTHAL, T. D. (1993). Social anxiety and latency of response to startle stimuli. Journal of Research in Personality, 27, 1-14.

Cohen, M. E., Hoffman, H. S., \& StitT, C. L. (1981). Sensory magnitude estimation in the context of reflex modification. Journal of Experimental Psychology: Human Perception \& Performance, 7, 13631370.

Dorthe, N. J., Blumenthal, T. D., Jason, D. R., \& Lantz, P. E. (1995). The use of next-of-kin in assessing handedness. Perceptual \& Motor Skills, 81, 203-208.

Elmasian, R., Galambos, R., \& Bernheim, A. (1980). Loudness enhancement and decrement in four paradigms. Journal of the Acoustical Society of America, 67, 601-607.

ENGEN, T. (1972). Psychophysics II. Scaling methods. In J. W. Kling \& L. A. Riggs (Eds.), Experimental psychology: Vol. I. Sensation and perception (3rd ed., pp. 47-86). New York: Holt, Rinehart \& Winston.

Gescheider, G. A. (1985). Psychophysics: Method, theory, and application (2nd ed.). Hillsdale, NJ: Erlbaum.

Graham, F. K. (1975). The more or less startling effects of weak prestimulation. Psychophysiology, 12, 238-248.

GrahaM, F. K. (1979). Distinguishing among orienting, defense, and startle reflexes. In H. D. Kimmel, E. H, van Olst, \& J. F. Orlebeke (Eds.), The orienting reflex in humans (pp. 137-167). Hillsdale, NJ: Erlbaum.

Norris, C. M., \& Blumenthal, T. D. (1995). Evidence for the protection of preattentive processing during inhibition of the acoustic startle response. Psychophysiology, 32, S57.

Perlstein, W. M., Fiorito, E., Simons, \& Graham, F. K. (1993). Lead stimulation effects on reflex blink, exogenous brain potentials, and loudness judgments. Psychophysiology, 30, 347-358.

Silverstein, L. D., Graham, F. K., \& Bohlin, G. (1981). Selective attention effects on the reflex blink. Psychophysiology, 18, 240-247.

YamadA, F. (1983). Effects of intensity, duration, and rise and fall times, of the reflex evoking sound upon auditory evoked EMG of orbicularis oculi and subjective estimation of startle. Japanese Journal of Physiological Psychology \& Psychophysiology, 1, 11-18.

\section{NOTE}

1. We would like to thank E. Krauter for suggesting this idea.

(Manuscript received June 7, 1994; revision accepted for publication June 2, 1995.) 